\title{
Rates of Joint Replacement Surgery in New Zealand, 1999-2015: A Comparison of Rheumatoid Arthritis and Osteoarthritis
}

\author{
Lisa K. Stamp, Janine Haslett, Peter Chapman, John O’Donnell, Rafi Raja, Alastair Rothwell, \\ Christopher Frampton, and Gary Hooper
}

\begin{abstract}
Objective. To determine rates of joint replacement for people with rheumatoid arthritis (RA) and osteoarthritis (OA) and to examine the characteristics of those receiving elbow replacements.

Methods. Data were extracted from the New Zealand Joint Registry from 1999 to 2015 and annual rates calculated.

Results. Rates of joint replacement increased over time for OA but not RA. Elbow replacement was the only procedure performed more commonly in RA.

Conclusion. There has been a substantial increase in joint replacement for OA in New Zealand. For RA, where access to biologics has been limited to those with erosions, joint replacement rates have not declined, with the exception of elbow replacements. (First Release October 15 2017; J Rheumatol 2017;44:1823-7; doi:10.3899/jrheum.170551)
\end{abstract}

Key Indexing Terms: RHEUMATOID ARTHRITIS

\section{OSTEOARTHRITIS}

SURGERY

Poorly controlled rheumatoid arthritis (RA) may lead to secondary osteoarthritis (OA), an indicator for joint replacement. In a US RA cohort from 1974, joint replacement was reported in $25 \%$ within 20 years of disease onset ${ }^{1}$. The last 10-15 years has seen earlier, more intensive use of disease-modifying antirheumatic drugs (DMARD) with treatto-target strategies the standard of care. Introduction of biological (b)DMARD has provided effective disease control for many, including those who have failed traditional DMARD treatment.

Studies in a number of countries have reported a reduction in joint replacements with increased intensity of medical therapy $y^{2,3,4,5}$. This has not been a universal finding, with joint replacement rates decreasing minimally in people with RA

From the Department of Medicine and Department of Orthopaedics, University of Otago; Department of Rheumatology, Immunology and Allergy, Christchurch Hospital, Christchurch, New Zealand.

This study was funded by the Canterbury Arthritis Society Trust.

L.K. Stamp, FRACP, PhD, Department of Medicine, University of Otago, and Department of Rheumatology, Immunology and Allergy, Christchurch Hospital; J. Haslett, Research Coordinator, Department of Medicine, University of Otago; P. Chapman, FRACP, MD, Department of Rheumatology, Immunology and Allergy, Christchurch Hospital; J. O'Donnell, FRACP, Department of Rheumatology, Immunology and Allergy, Christchurch Hospital; R. Raja, FRACP, Department of Rheumatology, Immunology and Allergy, Christchurch Hospital; A. Rothwell, Orthopedic Surgeon, FRACS FNZOA; C. Frampton, Biostatistician, PhD, Department of Medicine, University of Otago; G. Hooper, Orthopedic Surgeon, FRACS FRNZOA, Department of Orthopaedics, University of Otago.

Address correspondence to Professor L.K. Stamp, Department of Medicine, University of Otago, Christchurch, P.O. Box 4345,

Christchurch, New Zealand.E-mail: lisa.stamp@cdhb.health.nz

Accepted for publication August 4, 2017. between 1991 and $2005^{6}$, and a reduction in small but not large joint replacements ${ }^{7}$.

In New Zealand (NZ), bDMARD have been funded since 2006 but until 2017 were only available for people with RA who had radiographic evidence of erosions, had failed multiple DMARD, including triple therapy, and had evidence of active $\mathrm{RA}^{8}$. This compares to other countries where radiographic erosion is not a prerequisite. It is therefore possible that rates of joint replacement in NZ have remained static or increased.

There have been concerns that patients with RA, especially those receiving bDMARD, may be more likely to have complications, particularly infection, with joint replacement. A higher risk of dislocation after hip replacement and infection after knee replacement compared to those with OA has been reported ${ }^{9}$. While the role of anti-tumor necrosis factor agents in prosthetic joint infection remains unclear, some studies have suggested an increased risk especially when combined with steroids ${ }^{10}$. Discontinuation of biologics has been recommended at least 1 dosing interval prior to joint replacement to reduce the potential for infection ${ }^{11}$.

The aims of our study were to determine trends in joint replacement for people with RA compared to OA in NZ and to examine the clinical characteristics of those with RA undergoing elbow replacement. This procedure is usually undertaken for inflammatory rheumatic diseases rather than primary osteoarthritis.

\section{MATERIALS AND METHODS}

Our study was approved by the NZ Health and Disability Ethics Committee

Personal non-commercial use only. The Journal of Rheumatology Copyright $\subset$ 2017. All rights reserved. 
(15/STH/30). People with RA and OA were identified from the NZ Joint Registry (NZJR) from 1999 to 2015 . NZJR is a national register of all joint replacements, public and private, throughout NZ since 1999. Details of the underlying diagnosis including RA and OA are identified by the treating surgeon.

People with RA from Christchurch, NZ, who received an elbow replacement from 2000 to 2013 were identified from the NZJR (cases). For each case, 2 RA controls residing in Christchurch were identified from the Christchurch Hospital Rheumatology Department database or the NZJR. Controls were matched on age \pm 5 years at date of joint replacement, sex, and date of diagnosis \pm 5 years. One control had no previous joint replacements and 1 control had a hip replacement.

Medical records were reviewed to collect data including demographics, current and previous DMARD use, previous joint replacement, oral or intraarticular steroid, radiographic erosions, and complications defined as infection, dislocation, loosening, or revision.

Statistics. The NZ population $>15$ years old was obtained from Statistics NZ Census data, which were available for 1996, 2001, 2006, and $2013^{12}$. Between census dates the population was estimated using linear interpolation. From the NZJR data, rates of hip, knee, ankle, shoulder, and elbow replacement for OA and RA per 100,000 population for each indication were calculated. Demographic and clinical features were compared between individually matched groups using paired t tests, Wilcoxon signed rank tests, and McNemar's chi-squared tests as appropriate. Two-sided p value $<0.05$ was taken to indicate statistical significance.

\section{RESULTS}

Rates of joint replacement over time. Hip and knee were the most common joint replacements. The total number of hip, knee, and shoulder replacements increased between 1999 and 2015. Only small numbers of elbow and ankle replacements were undertaken (Figure 1).

With the exception of elbow replacements, the rates of joint replacement were higher for OA compared to RA (Figure 1). The increase in hip, knee, shoulder, and ankle replacements over time was due to an increase in joint replacements for OA. Rates of hip, knee, shoulder, and ankle replacement for RA remained stable over time. Rates of elbow replacement for RA decreased from 2008 to 2013 (Figure 1e).

Elbow replacements. Between 2000 and 2013 a total of 59 elbow replacements were undertaken in 49 individuals in Christchurch. Twelve individuals did not reside in Christchurch so no further details could be obtained, and 3 did not have RA. For those individuals with bilateral elbow replacements, data were collected for the first elbow replaced. Thus a total of 34 individuals with an elbow replacement were included. The number of elbow replacements per year was low (0-6; Figure 1f).

The majority of people with RA undergoing elbow replacement were female with mean (SD) age of 64.3 (11.8) years (Table 1). Compared to RA patients without previous joint replacement, those with an elbow replacement were more likely to have received oral steroid prior to surgery and were more likely to have had intraarticular steroid injections, but were less likely to have rheumatoid nodules (Table 1). Compared to those with a hip replacement, those with elbow replacement had a significantly longer disease duration (27.4 \pm 11.5 vs $24.3 \pm 10.9$ years; $p=0.02$ ) and a higher mean number of DMARD prior to surgery (Table 1). Of those with an elbow replacement, $73 \%$ had a previous joint replacement, with mean (range) of 2.18 (0-7) joints replaced prior to elbow replacement. Compared to those with a hip replacement, individuals with an elbow replacement had a higher mean number of previous joint replacements (2.18 vs 1.15 ; $\mathrm{p}=0.02$ ). Overall rates of biologic use were low (Table 1). There were significantly fewer complications in those with an elbow replacement compared to matched controls with a hip replacement ( $1 / 34$ vs $8 / 34 ; p=0.02)$.

\section{DISCUSSION}

While there has been an increase in joint replacement for OA, rates have remained stable over time for RA. Despite more intensive therapeutic strategies and the introduction of bDMARD in 2006, there has been no obvious decline in rates of joint replacement, with the exception of elbow replacements, for RA to date. Overall, the rate of joint replacement in people with RA in the United States has been reported to be $\sim 4.6 / 100,000$, similar to the rates observed in our study ${ }^{6}$.

There are a number of reasons why joint replacement rates for RA have not declined. First, these rates are relatively low because access to joint replacement in NZ in the publicly funded healthcare system, where the majority of people with RA are managed, is restricted to those with severe functional limitation. This is indicated by the long disease duration (>24 yrs) for those undergoing elbow and hip replacement. Second, joint damage usually occurs early in the course of the disease ${ }^{13}$. Suppressing RA synovitis will not prevent progression of established OA. The treat-to-target approach was not developed until the late 1990s, thus it may be another $10-15$ years before any reductions may be evident in those patients with RA disease durations $>20$ years. Further, the introduction of biologics in NZ occurred in 2006 with access to adalimumab. Broadening of access with funding of etanercept did not occur until 2009. Given the relatively strict eligibility criteria for bDMARD, it is possible that this use of bDMARD (once joints have radiographic damage) may lessen the anticipated decline in rates of joint replacement. Given the restricted access, the rates of biologic use in this cohort are low when compared to some other countries such as the United States ${ }^{14}$.

Elbow replacements, while infrequently undertaken, are the only joint replacement procedures where surgery rates are higher for RA compared to OA. This is in keeping with data from the Scottish Arthroplasty project, where inflammatory arthritis was the indication for elbow replacement in $79 \%$ of cases $^{15}$. However, in Scotland, there was a significant reduction in elbow replacements over 18 years predominantly due to a reduction in elbow replacements for inflammatory arthritis ${ }^{15}$. It is important to note that elbow replacement is a technically demanding procedure and in general, surgery is deferred as long as possible in comparison to hip and knee replacements.

Personal non-commercial use only. The Journal of Rheumatology Copyright @ 2017 . All rights reserved. 
a) Hips

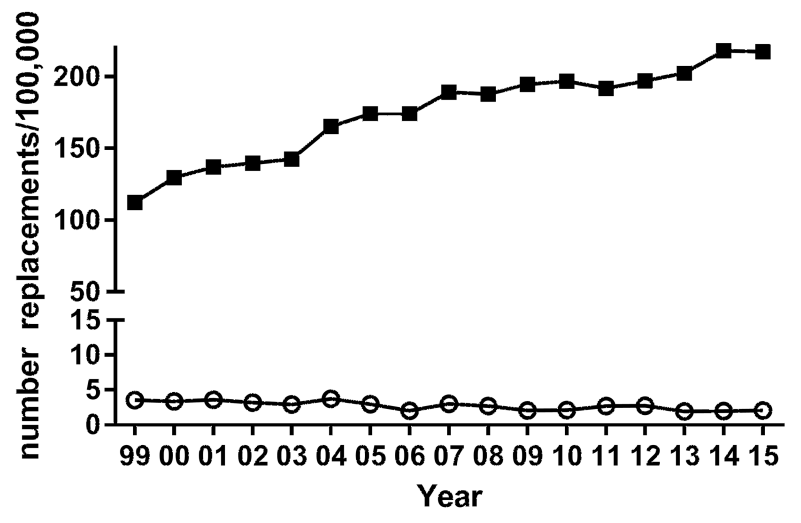

c) Shoulders

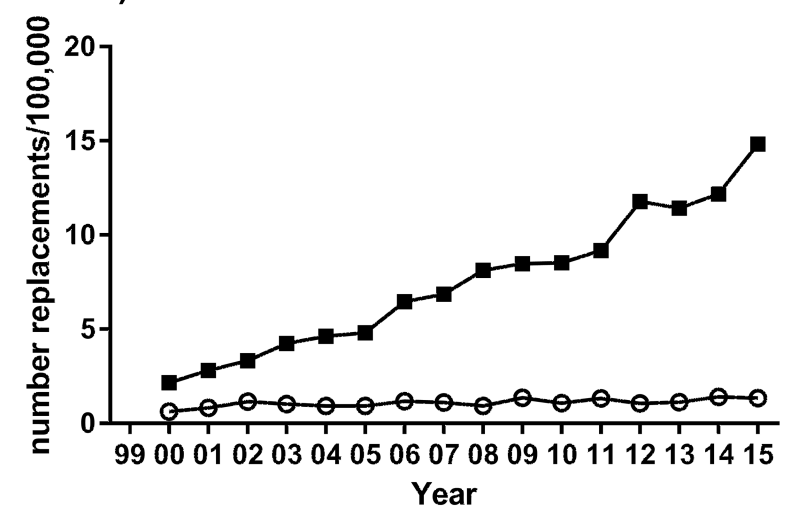

e) Elbows

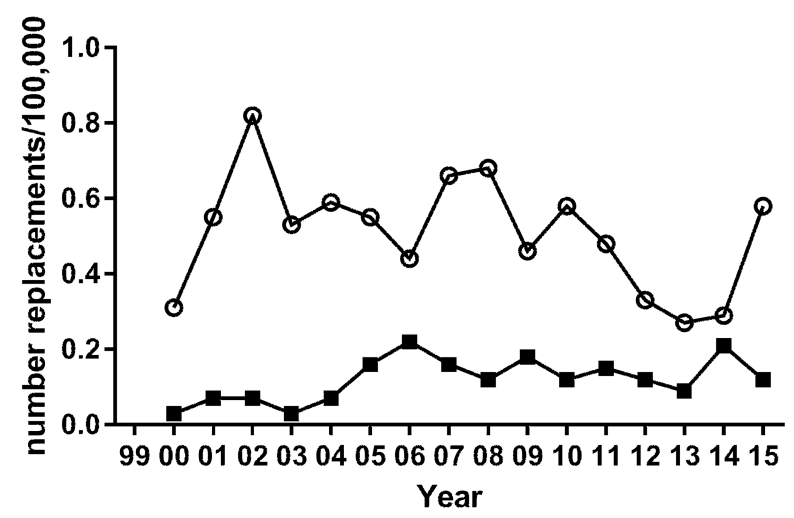

b) Knees

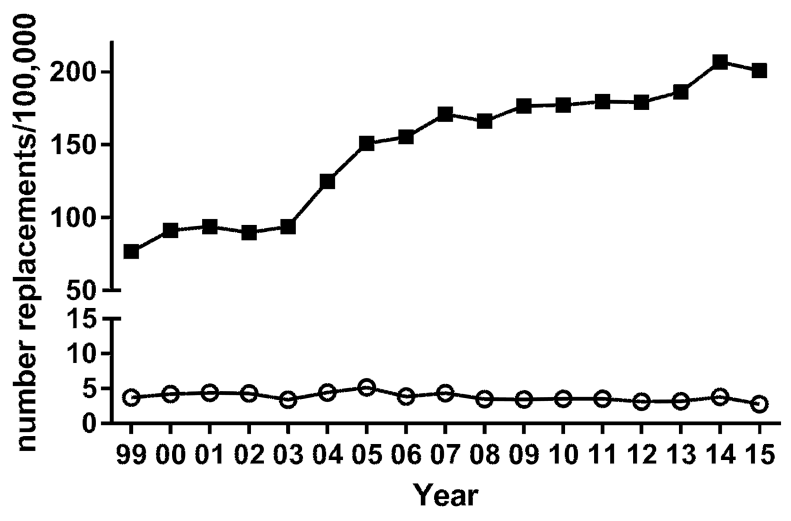

d) Ankles

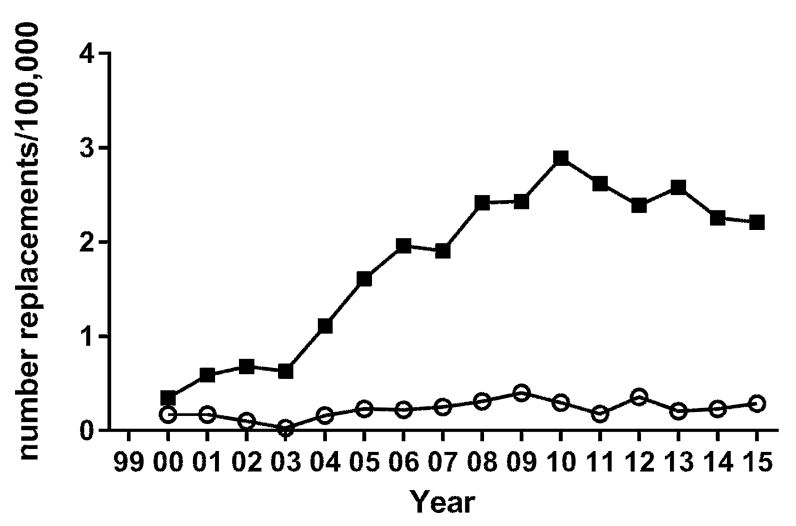

f) Elbows

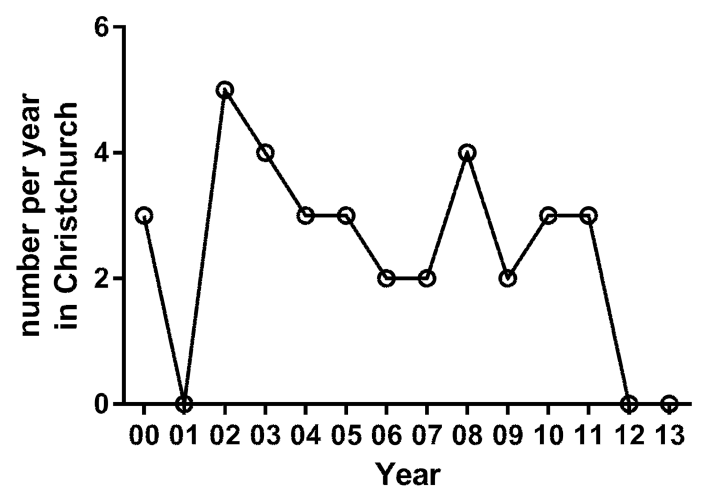

Figure 1. Number of joint replacements per 100,000 per year in New Zealand for people with RA and OA for (a) hips, (b) knees, (c) shoulders, (d) ankles, (e) elbows, and (f) no. elbow replacements per year in Christchurch. Lines with black squares indicate OA, and lines with open circles indicate RA. RA: rheumatoid arthritis; OA: osteoarthritis.

The data presented in our study suggest that those individuals undergoing an elbow replacement may have had more severe RA with more use of steroids prior to surgery. There may have been some selection bias in the comparisons between those with elbow and hip replacements. Although cases and controls were matched on disease duration, hip replacement data are available from 1999, whereas elbow replacements have only been done since 2000 .

Despite the high use of DMARD and steroids at the time of surgery, complications were rare. There were more complications in those undergoing hip replacement. In particular, recurrent dislocations were common but infections and

\section{Personal non-commercial use only. The Journal of Rheumatology Copyright @ 2017 . All rights reserved.}


Table 1. Comparison of clinical features between those with and without an elbow replacement. Data are n (\%) unless otherwise indicated.

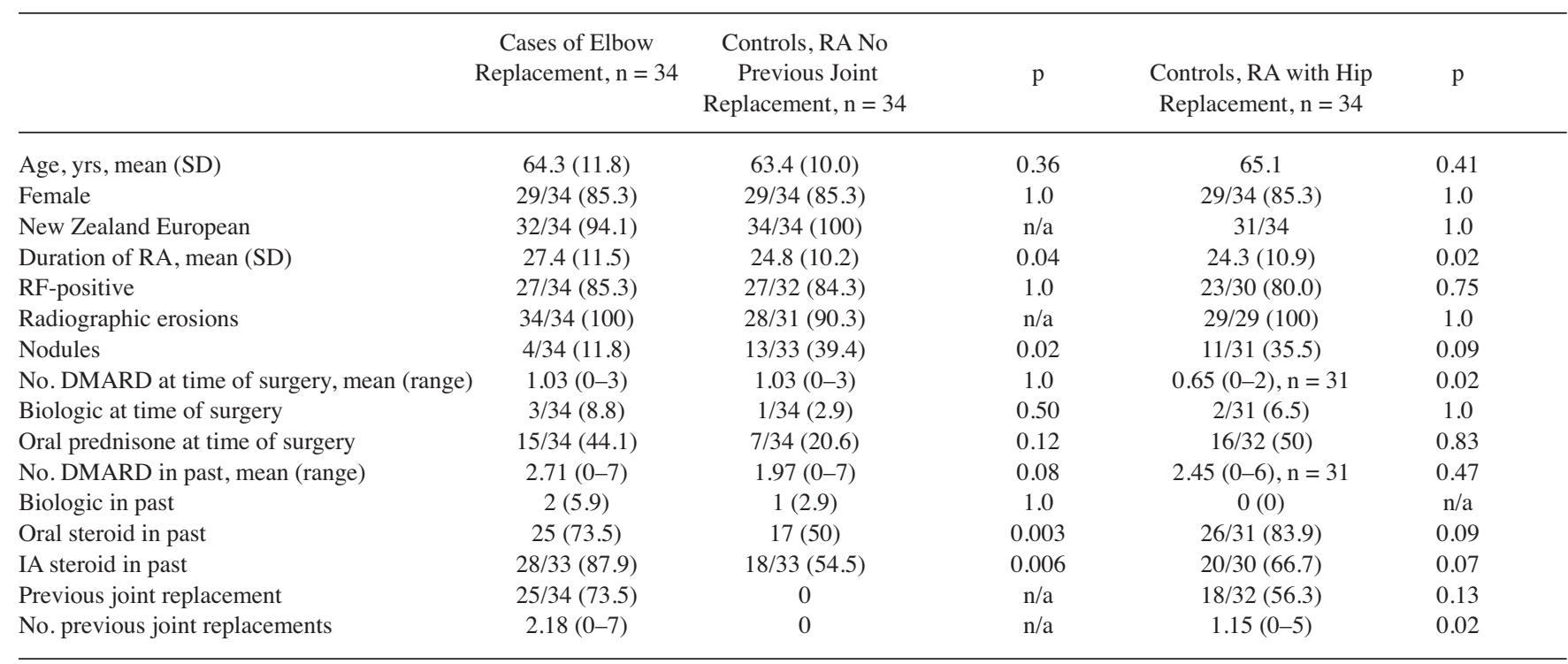

RA: rheumatoid arthritis; RF: rheumatoid factor; DMARD: disease-modifying antirheumatic drugs; IA: intraarticular; n/a: not applicable.

wound dehiscence were uncommon. A higher rate of hip dislocations in people with RA compared to those with OA has been noted previously 9 .

There are some limitations to our study. Importantly, the diagnosis of RA and OA in the NZJR is determined by the treating surgeon and it was not possible to verify the diagnosis for the entire national database. The diagnosis has not been formally validated and from review of the medical records, 3/49 (6\%) with elbow replacements did not have $\mathrm{RA}$. It is recognized that such databases can result in misdiagnosis ${ }^{16,17}$. Second, despite treat-to-target recommendations, it was not possible for us to determine whether this recommendation was followed by rheumatologists throughout NZ. Finally, while we have not undertaken any analysis by region and access to rheumatology care, marked variation in joint replacement rates has been previously reported across different locations, and in areas with poor access to specialist rheumatology care there are lower rates of joint replacement ${ }^{18}$.

Rates of joint replacement have not declined for RA in NZ where access to biologics has been limited to those with erosions, with the exception of elbows.

\section{ACKNOWLEDGMENT}

We are grateful to the New Zealand Joint Registry and Toni Hobbs.

\section{REFERENCES}

1. Wolfe F, Zwillich S. The long-term outcomes of rheumatoid arthritis. A 23-year prospective, longitudinal study of total joint replacement and its predictors in 1,600 patients with rheumatoid arthritis. Arthritis Rheum 1998;41:1072-82.

2. Jämsen E, Virta L, Hakala M, Kauppi M, Malmivaara A, Lehto M. The decline in joint replacement surgery in rheumatoid arthritis is associated with a concomitant increase in the intensity of anti-rheumatic therapy: a nationwide register-based study from 1995 through 2010. Acta Ortho 2013;84:331-7.

3. Hekmat K, Jacobsson L, Nilsson J, Petersson I, Robertsson O, Garellick G, et al. Decrease in the incidence of total hip arthroplasties in patients with rheumatoid arthritis - results from a well defined population in south Sweden. Arthritis Res Ther 2011;13:R67.

4. Louie G, Ward M. Changes in the rates of joint surgery among patients with rheumatoid arthritis in California, 1983-2007. Ann Rheum Dis 2010;69:868-71.

5. Harty L, O'Toole G, Fitzgerald O. Profound reduction in hospital admissions and musculoskeletal surgical procedures for rheumatoid arthritis with concurrent changes in clinical practice (1995-2010). Rheumatology 2015;54:666-71.

6. Mertelsmann-Voss C, Lyman S, Pan T, Goodman S, Figgie M, Mandl L. US trends in rates of arthroplasty for inflammatory arthritis including rheumatoid arthritis, juvenile idiopathic arthritis, and spondyloarthritis. Arthritis Rheumatol 2014;66:1432-9.

7. Nikiphorou E, Carpenter L, Morris S, MacGregor A, Dixey J, Kiely $\mathrm{P}$, et al. Hand and foot surgery rates in rheumatoid arthritis have declined from 1986 to 2011, but large-joint replacement rates remain unchanged: results from two UK inception cohorts. Arthritis Rheumatol 2014;66:1081-9.

8. PHARMAC. Adalimumab Special Authority. www.pharmac.govt.nz/2017/10/01/SA1621.pdf

9. Ravi B, Croxford R, Hollands S, Paterson J, Bogoch E, Kreder H, et al. Increased risk of complications following total joint arthroplasty in patients with rheumatoid arthritis. Arthritis Rheumatol 2014;66:254-63.

10. Gilson M, Gossec L, Mariette X, Gherissi D, Guyot M, Berthelot J, et al. Risk factors for total joint arthroplasty infection in patients receiving tumor necrosis factor $\alpha$-blockers: a case-control study. Arthritis Res Ther 2010;12:R145.

11. Goodman SM, Springer B, Guyatt G, Abdel MP, Dasa V, George M, et al. 2017 American College of Rheumatology/American Association of Hip and Knee Surgeons guideline for the perioperative management of antirheumatic medication in patients with rheumatic diseases undergoing elective total hip or total knee arthroplasty. Arthritis Rheumatol 2017;69:1538-51. 
12. Statistics New Zealand. 2013 Census QuickStats about national highlights. [Internet. Accessed September 11, 2017.] Available from: www.stats.govt.nz/ /media/Statistics/Census/ 2013\%20Census/profile-and-summary-reports/ quickstats-about-national-highlights/qs-nat-high-tables.xls

13. Fuchs H, Kaye J, Callahan L, Nance E, Pincus T. Evidence of significant radiographic damage in rheumatoid arthritis within the first 2 years of disease. J Rheumatol 1989;16:585-91.

14. Zhang J, Xie F, Delzell E, Chen L, Kilgore M, Yun H, et al. Trends in the use of biologic agents among rheumatoid arthritis patients enrolled in the US medicare program. Arthritis Care Res 2013;65:1743-51.

15. Jenkins P, Watts A, Norwood T, Duckworth A, Rymaszewski L, McEachan J. Total elbow replacement: outcome of 1,146 arthroplasties from the Scottish Arthroplasty Project. Acta Orthop 2013;84:119-23.

16. Gabriel S. The sensitivity and specificity of computerized databases for the diagnosis of rheumatoid arthritis. Arthritis Rheum 1994;37:821-3.

17. Kim S, Solomon D. Use of administrative claims data for comparative effectiveness research of rheumatoid arthritis treatments. Arthritis Res Ther 2011;13:129.

18. Harcombe H, Davie G, Derrett S, Abbott H, Gwynne-Jones D. Equity of publicly-funded hip and knee joint replacement surgery in New Zealand: results from a national observational study. N Z Med J 2016;129:8-18. 